\title{
Virtual laboratories on energy management systems: the Hybrid Electric Vehicle case
}

\author{
Alba Escolà, Arnau Dòria-Cerezo ${ }^{*, * *}$ Ramon Costa-Castelló ${ }^{* *, * * *}$ \\ * Department d'Enginyeria Elèctrica (EE) \\ ** Departament d'Enginyeria de Sistemes, Automàtica i Informàtica \\ Industrial (ESAII) \\ *** Institut d'Organització i Control de Sistemes Industrials(IOC), \\ Universitat Politècnica de Catalunya (UPC), Barcelona, 08028 Spain \\ (e-mail: \{arnau.doria,ramon.costa\}@upc.edu)
}

\begin{abstract}
Due to environment and social needs, energy management is becoming a key easy which must be transversely introduced to engineering students. Unfortunately most of this topics are difficult to analyze in traditional laboratories, this paper presents a virtual laboratory on Hybrid Electrical Vehicles specially designed to illustrate the power flow and the energy management algorithms involved in this type of device. The paper describes the models behind the interactive virtual laboratory, the different configurations which can be taken into account and the implementation technology.
\end{abstract}

Keywords: interactivity, virtual laboratory, hybrid electrical vehicle, energy management

\section{INTRODUCTION}

Environmental problems and the increasing energy consumption have make that social actors, including engineers, turn they attention into energy generation/transformation/consumption processes. A generic goal in all these processes is to optimize all components and methods in order to reduce energy consumption while minimizing generated residual, and control theory can play a key role in the energy management processes. Introducing these transversal knowledge in engineering studies is a challenging goal that must be addressed by academics.

A tool that has proved efficient in order to shortcut and simplify the access to new concepts and technologies is interactivity [Dormido et al., 2005, Sánchez et al., 2004]. Interactivity allows to understand qualitatively the influence of parameters in the system behavior without the need of an in-depth knowledge of a certain subject.

Interactive Virtual Laboratories (IVL) are software developments which emulate a real system. This environments allow to obtain stimuli and feelings similar to the ones obtained in a hands-off laboratory with the only need of a personal computer. In order to achieve the desired pedagogical goals IVL should be complemented with a documentation set and a set of guided exercises to be develop over the IVL. This educational material help students through the sessions and proposes appropriate experiments in order to understand better the behavior of the system.

A field which is becoming very active in the introduction and design of new energy management technologies is the automotive field, concretely a lot of work is being done in order to obtain and optimize Hybrid Electric Vehicles
(HEV). This paper describes an IVL especially designed to introduce students in energy flow and energy management in $\mathrm{HEV}$.

HEV have been largely studied in the last years [Ehsani et al., 2004, Miller, 2004]. They combine the traditional Internal Combustion Engine (ICE) with an electrical system. The major achievement of this system is regenerative breaking introduction, i.e., the capability of storing the energy used to break the vehicle into a energy storage device (batteries or ultra-capacitors mainly). Besides, in some topologies the electrical system allows to modify the operation point of the ICE in order to set it in an optimal one.

$\mathrm{HEV}$ can be classified in two large families, depending on the topology: series and parallel topologies. Fig. 1 shows these two topologies. In series connection there's no direct connection between the ICE and the mechanical traction system while in the parallel connection both the electrical motor and the ICE have direct connection to the mechanical traction system. Main advantages of the series configuration is the simplicity of the system and the mechanical decoupling between the ICE and the wheels, and consequently, the ability to regulate the ICE speed independently to the velocity of the vehicle. Disadvantages includes the use of two electrical machines and that the electrical motor must manage all mechanical traction. In the opposite, the parallel configuration benefits to the fact that the power of the vehicle is the sum of the ICE and the electrical system and the smaller size of the whole propulsion system. The coupling of the mechanical and electrical parts and the direct relationship between ICE and the vehicle speed are the main disadvantages of this configuration. 

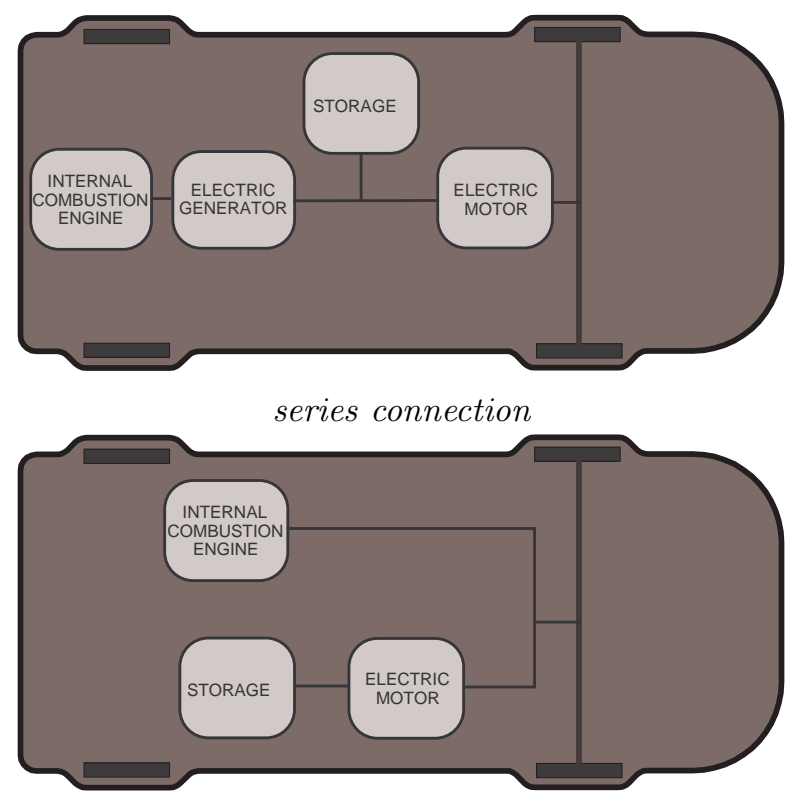

parallel connection

Fig. 1. Series and parallel configurations of an HEV.

Although more topologies are also possible combining both described basic topologies, [Chan, 2002], in the described IVL only the series and parallel configurations are considered.

This paper is organized as follows. Section 2 contains the developed model of the HEV. Basic control algorithms, as well as the energy management elements, are described in Section 3. In order to compare different strategies and topology a standard speed profile is adopted, Section 4 introduces the New European Driving Cycle (NEDC) speed profile. The IVL is presented in Section 5, including a brief description of its possibilities. Finally, Conclusions are stated in Section 6.

\section{HEV MODEL}

\begin{tabular}{|c|c|c|c|}
\hline A & B & C & Configuration \\
\hline \hline $\begin{array}{c}\text { off } \\
\text { on }\end{array}$ & $\begin{array}{c}\text { on } \\
\text { off }\end{array}$ & $\begin{array}{c}\text { on } \\
\text { on }\end{array}$ & Parallel \\
on & on & offies & Conventional \\
\hline
\end{tabular}

Table 1. Architecture selection table.

This section describes the mathematical model which has been developed to simulate the HEV behavior. This model can be parameterized in order to reproduce most relevant topologies currently in HEV. The model also allows to emulate a conventional vehicle with only an ICE propulsion system.

The system is composed by different parts: the Internal Combustion Engine (ICE), two electric machines (so called generator and motor), three power converters, storage elements (batteries and ultra-capacitors), electrical loads (such as air conditioning, lights...), mechanical transmission, and the car dynamics. All the electrical parts are interconnected by means of a common high voltage point (DC bus). Fig. 2 shows how all these parts and interconnected.
Combining the switches $\mathrm{A}, \mathrm{B}, \mathrm{C}$ three topologies can be reproduced. In table 1 concrete topology and related switch configurations are described.

It is important to emphasize that the goal of a IVL is to qualitatively reproduce the behavior of a certain system in a graphical and interactive manner, as a consequence the behavior of all these parts have been modelled by very simple equations, most of them reduced to the steady state behavior in order to obtain interactive results. Although models are simple, they have proved good enough to visualize most energetic phenomena and the relevance of each element in the energy management process.

\subsection{The DC bus}

All electrical components (power converters, electric loads, the battery and the ultra-capacitor) are connected through a DC bus. All elements connected in the DC bus share the same voltage, $V_{b a t}$, which in the model is fixed by the batteries in order to assure causality (subsection 2.4). This connection is modelled using the Kirchhoff laws,

$$
i_{p g} \cdot B=i_{b a t}+i_{p c}+i_{l}+i_{p m},
$$

where $i_{p g}$ is the current given by the generator power converter, $i_{b a t}$ is the current from the batteries, $i_{p c}$ is the current coming from the ultra-capacitors power converter, $i_{l}$ is the current required by the loads and $i_{p m}$ is the current required from the electrical motor.

\subsection{Electrical machines}

Different from conventional vehicles, electrical machines play an important role in HEV. Permanent magnet motors, induction motors, and recently switched reluctance machines, are considered the most likely candidates for the propulsion system for an HEV [Ehsani et al., 2007]. Since the dynamics of the electrical machines are faster that the vehicle ones, they are reduced to a simple static relationships, i.e.,

$$
\begin{aligned}
V_{k} & =k_{k} \cdot \omega_{k} \\
\tau_{k} & =k_{k} \cdot i_{k},
\end{aligned}
$$

where $V_{k}$ is the power converter voltage in the electrical machine part, $i_{k}$ is the current required by the electrical machines, $\tau_{k}$ the electrical torque and $\omega_{k}$ the mechanical speed of each machine (where the subindex $k$ is $g$ or $m$ depending if the electrical machine works as generator or motor respectively).

\subsection{Power converters}

Power converters are switched electronic devices which allow to regulate the electrical power flow in the system. The power flow in the power converter is indicated by means of a control signal. In the proposed scheme the power converter allows to control the energy flow to the electrical machines and the ultra-capacitors (Section 3 ). The simplified static model for the power converter (neglecting the losses) is

$$
\begin{aligned}
V_{b a t} & =n V_{k} \\
i_{p k} & =\frac{1}{n} i_{k},
\end{aligned}
$$




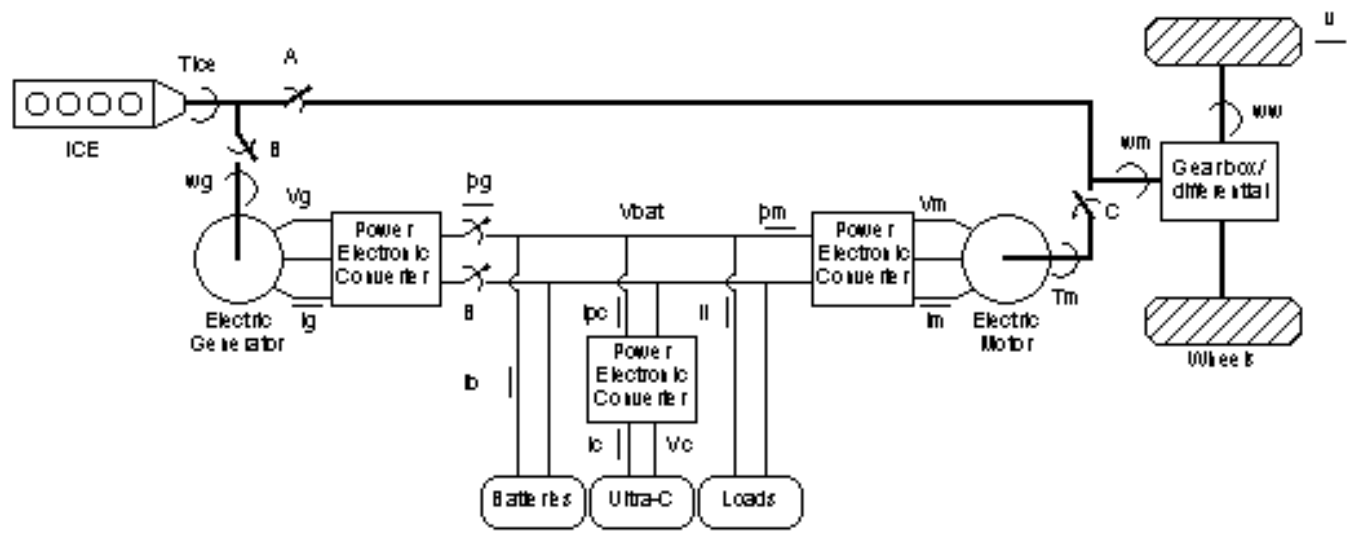

Fig. 2. Scheme of the proposed model, which includes can be configured as a conventional, series HEV or parallel HEV topology depending on the switch $(\mathrm{A}, \mathrm{B}, \mathrm{C})$ configuration.

where $n$ is the ratio of the voltage input/output, $V_{k}$ and $i_{k}$ are voltages and currents, and subindex $p k$ refers to the DC bus side of the converter and $k$ to the element side (generator, $g$, ultra-capacitor, $c$, or motor, $m$ ).

\subsection{Storage elements}

Batteries and ultra-capacitors are included in the HEV as energy storagement elements[Burke, 2007]. Only the energy in the ultra-capacitor is managed, while the energy stored in the batteries comes from the power balance of the system.

Although batteries are complex systems which include electro-chemical relations[Miller, 2004], most relevant battery dynamics can be described using the State Of Charge $(S O C)$ variable. $S O C$ can be obtained integrating the battery output current,

$$
\operatorname{SOC}(t)=\frac{1}{Q} \int_{0}^{t} i_{\text {bat }}(\tau) \mathrm{d} \tau,
$$

where $Q$ stands for the battery capacity. In a $\mathrm{NiMH}$ battery[Miller, 2004], the battery output voltage, $V_{b a t}$, is computed using the following algebraic equation:

$$
V_{b a t}=b_{1}\left(S O C-b_{2}\right)^{3}+V_{b a t}^{n o m},
$$

where $b_{1}, b_{2}$ are technological parameters depending on the concrete battery, and $V_{b a t}^{\text {nom }}$ is the battery nominal voltage.

Ultra-capacitors have been modelled as a current controlled capacitor. Ideally, the dynamics of a capacitor is

$$
\frac{\mathrm{d} V_{c}}{\mathrm{~d} t}=\frac{1}{C} i_{c}
$$

where $V_{c}$ is the ultra-capacitor voltage, $C$ is the capacitance and the $i_{c}$ current is controlled through the power converter.

Although similar in their generic behavior, ultracapacitors and batteries have some important diferences, especially those related with the capacitance and the instantaneous power they can handle [Ehsani et al., 2004, 2007].

\subsection{Electrical loads}

Current vehicles contain a number of comfort components such as conditioning system, illumination, or music equipment between others. All these components require electrical power and in some case have a great influence in the vehicle power flow. Although some of they may have relevant dynamic behavior from the energy management point of view they can be considered as dissipative components which can be characterized by their power consumption. In the proposed model the effect of these components is modelled as resistive load with a certain power consumption:

$$
P_{\text {load }}=P_{\text {light }}+P_{\text {radio }}+P_{\text {air }}
$$

where $P_{\text {light }}, P_{\text {radio }}$ and $P_{\text {air }}$ represent the power due to illumination, music and air conditioned respectively. From this power it is possible to obtain its current:

$$
i_{l}=\frac{P_{\text {load }}}{V_{\text {bat }}} .
$$

The laboratory contains a table of most popular consumption elements[Shen et al., 2003] which is used during the simulation.

\subsection{ICE model and fuel consumption}

The ICE has been considered as an ideal torque source, $\tau_{I C E}$. Once fixed the required ICE power, $P_{I C E}$, from the power manager (see Section 3 ), the torque is computed by means of

$$
\tau_{I C E}=\frac{P_{I C E}}{\omega_{I C E}}
$$

where $\omega_{I C E}$ is the ICE mechanical speed. Note that depending on the selected HEV topology, $\omega_{I C E}$ corresponds to the mechanical transmission (parallel case) or the electrical generator (series case),

$$
\omega_{I C E}= \begin{cases}\omega_{m} & \text { if conventional or parallel } \\ \omega_{g} & \text { if series }\end{cases}
$$

Note that, in the series configuration, an extra degree of freedom is available, selecting $\omega_{I C E}$. This speed is usually set in an optimal value in terms of efficiency[Sciarretta and Guzzella, 2007]. Fig. 3 shows the used map of efficiency which corresponds to a $30 \mathrm{~kW}$ ICE. 


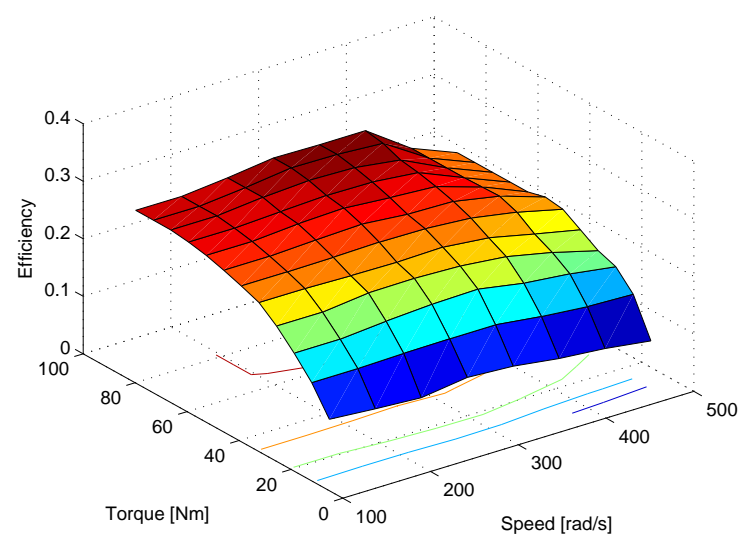

Fig. 3. ICE efficiency map of a 50Kw ICE.

The fuel consumption of the ICE, is also obtained as a function of the mechanical speed, $\omega_{I C E}$, and power, $P_{I C E}$. In Fig. 4 the fuel consumption of a $50 \mathrm{~kW}$ ICE is depicted.

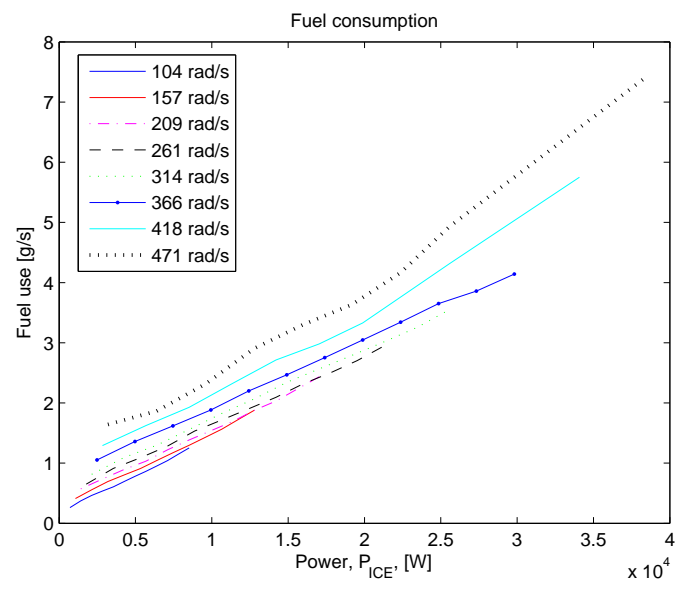

Fig. 4. ICE fuel consumption.

\subsection{Mechanical transmission and vehicle modelling}

The vehicle is modelled as a mass under the action of some external forces. From the second Newton's law, this dynamic system becomes

$$
m \frac{\mathrm{d} v}{\mathrm{~d} t}=F-F_{a}-F_{b}
$$

where $m$ is the mass vehicle, $v$ is the vehicle speed, while $F_{a}, F_{b}$ and $F$ are air damping, the breaking, and propulsion forces, respectively. The breaking force represents the force produced by the mechanical break and is determined by a simple supervisor algorithm detailed in Section 3.

The air damping force is given by[Ehsani et al., 2004]

$$
F_{a}=\frac{1}{2} \rho C_{d} A_{d} v^{2}
$$

where $\rho$ is the air density, $C_{d}$ is the aerodynamic coefficient and $A_{d}$ is the front area of the vehicle.

The propulsion effort is given by the generated torque after the transmission line $\tau_{w}$. Considering the mechanical transmission and gear box, the relationship between the mechanical power variables $\left(\omega_{m}, \tau_{I C E}\right.$ and $\left.\tau_{m}\right)$ and the dynamics vehicle $(F$ and $v)$ is given by

$$
\begin{aligned}
\omega_{m} & =\frac{g}{r} v \\
C \cdot \tau_{m}+A \cdot \tau_{I C E} & =\frac{r}{g} F
\end{aligned}
$$

where $r$ is the radius of the wheels and $g$ is the gear box ratio. Notice that $s$ appears in the torque equation (11) in order to take the concrete topology into account.

The gear box is automatically adapted depending on the speed.

\section{ENERGY MANAGEMENT}

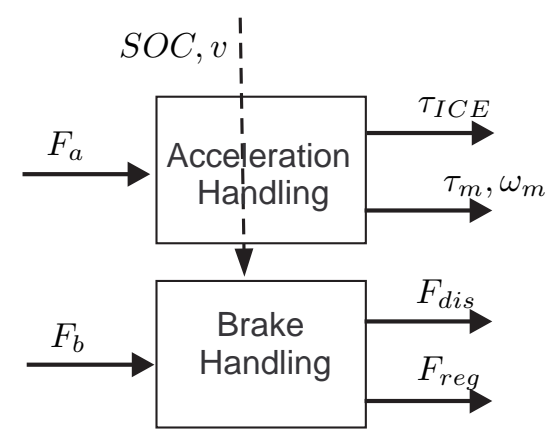

Fig. 5. Energy Management algorithm.

The main goal of any energy management algorithm [Sciarretta and Guzzella, 2007] is to minimize power consumption while following a certain predefined trajectory. As the trajectory is predefined needed power traction, $P_{\omega}$, is also predefined. As a consequence the only degree of freedom is how to generate the needed mechanical torque from the available power sources. Several approaches have been proposed in the literature[Sciarretta and Guzzella, 2007], some of them have been implemented in the described IVL. As an example, a very simple approach will be described. This approach consist in obtaining the desired ICE power as a percentage of the total power $P_{I C E}=K_{p} P_{\omega}$, where the power ratio, $K_{p}$, depends on the vehicle speed. As a simple approximation, $K_{p}$, is varying with the speed with a linear (or piecewise linear) function, see example in Fig. 8.

The other element which plays a key role in the energy management is regenerative braking[Ehsani et al., 2004], i.e. taking profit from kinetic energy during decelerating trajectories. Due to battery reduced current absorption possibilities, ultracapacitor play a key role in this process. Several management policies are possible, described IVL contains some of them. As an example, a simple one is described. Braking force needed to reduce the vehicle speed is decomposed in two parts, the first one is dissipated because it can not be stored in the system, $F_{d i s}$, and the second one is regenerated, $F_{\text {reg }}$, so $F_{b}=F_{\text {dis }}+F_{\text {reg }}$. This decomposition is not assumed constant, depends on two main elements:

- battery SOC. If the storage elements are full they can not store further energy so it is not convenient 
to generate regeneration force. In the opposite if the storage elements are empty they can absorb an important amount of energy.

- vehicle speed and acceleration. When the vehicle moves slowly the energy exchange will be small so storage elements will be able to absorb almost all the energy, on the contrary when the vehicle is moving fast energy exchange may be large, similarly if the speed variation is large the energy exchange will also be very important so most part of the energy will be dissipated

This tradeoff is tuned by means of a static curve which can be tuned in the IVL.

When simulated configuration contains ultra-capacitor, energy management become more complex due to the fact that two independent energy storage elements exits. Different from batteries ultra-capacitor can absorb an important amount in energy in small time, but their capacity is smaller than the battery one. These elements have also been included in the developed IVL.

Fig. 5 shows a complete scheme of the energy management algorithm. In the proposed IVL, $F_{a}$ and $F_{b}$ are automatically computed from the desired speed profile while in a real environment would be obtained form the accelerator and brake pedals respectively.

\section{STANDARD SPEED PROFILES}

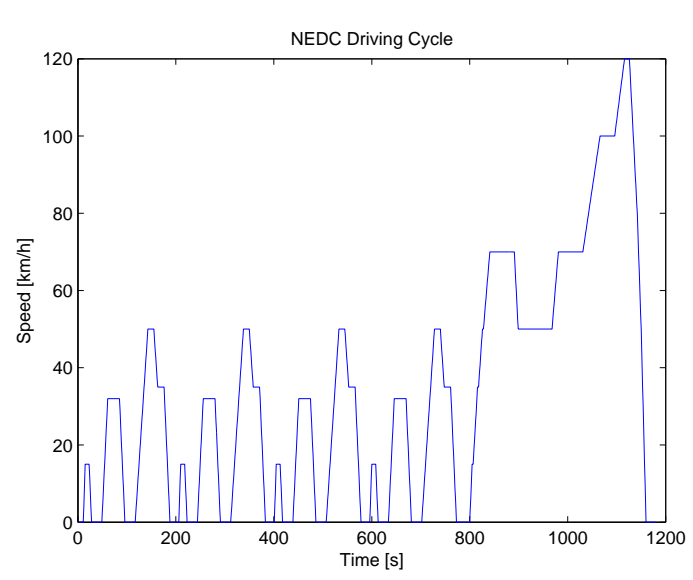

Fig. 6. Standard NEDC speed profile.

Currently there's no an standard energy management algorithm, in addition different energy management algorithms provides different results when applied under different circumstances. In order to compare different energy management algorithms and HEV topologies a set of standard speed profiles have been designed. These profiles take into account urban, road or highway driving, or a mixed case. One of the most used profiles in Europe is the Urban Driving Cycle (UDC, also known as ECE-15) and EUDC (Extra Urban Driving Cycle).

The ECE cycle is an urban driving cycle, also known as UDC. It was devised to represent city driving conditions, e.g. in Paris or Rome. It is characterized by low vehicle speed, low engine load, and low exhaust gas temperature. Extra Urban Driving Cycle (EUDC), simulating highway driving conditions. The maximum speed of the EUDC cycle is $120 \mathrm{~km} / \mathrm{h}$.

The default driving cycle included in the IVL is the New European Driving Cycle (NEDC), see Fig. 6. The NEDC contains four segments of UDC, and one of the EUDC. The EUDC segment has been added after the fourth ECE cycle to account for more aggressive, high speed driving modes.

\section{HEV VIRTUAL LAB DESCRIPTION}

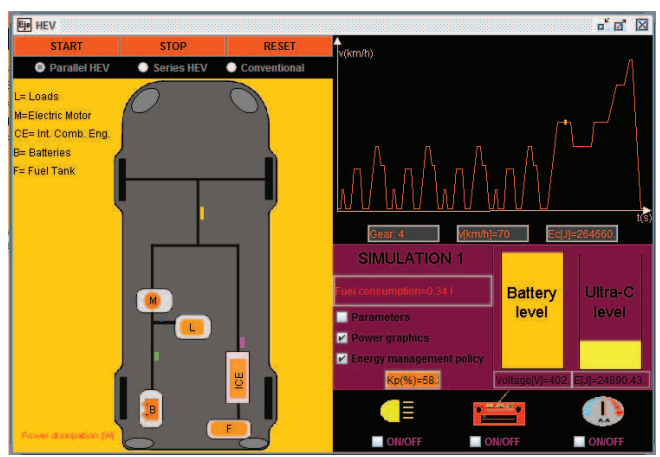

Fig. 7. Energy management algorithm GUI.

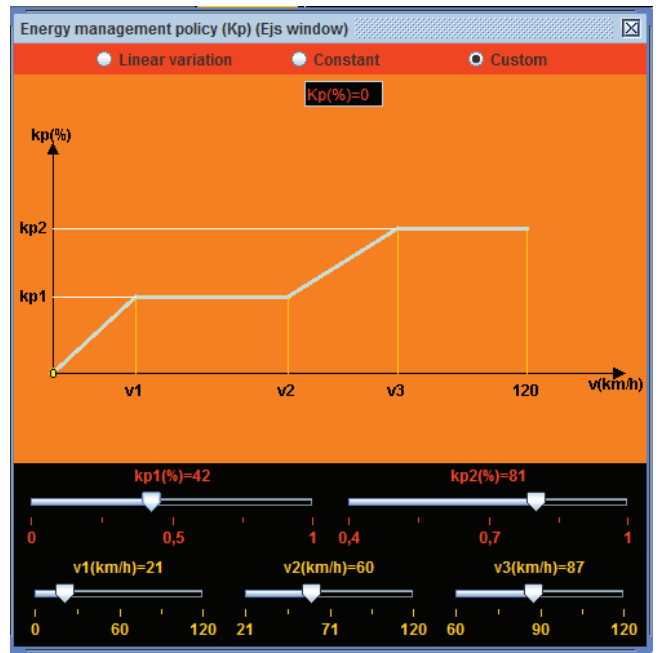

Fig. 8. Interactive window to select and costume the $K_{p}$ variable

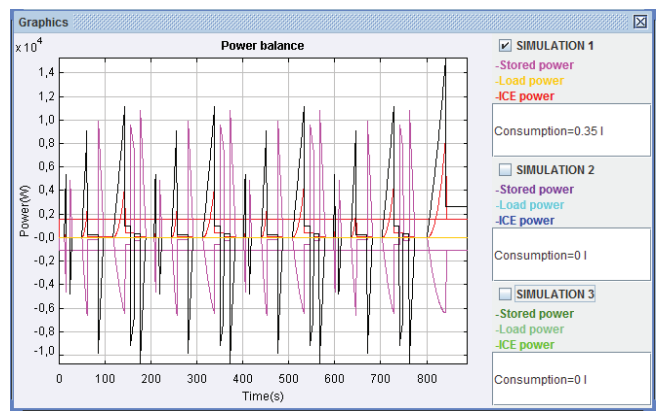

Fig. 9. Scope window with the power balance.

Presented IVL has been entirely developed using Easy Java Simulations, EJS [Esquembre, 2004, 2005]. Easy Java Simulations (EJS) is a free and open-source interactive 
tool developed to build IVL in a very simple and straightforward manner. In order to code IVL, EJS uses a model-view-control paradigm, in other words, the IVL laboratory is decomposed in three almost independent parts:

(1) Model: Variables and relations between these variables. This includes the differential and algebraic equations introduced in previous sections.

(2) View: The view corresponds to the virtual laboratory outputs. These outputs are constituted by different windows which contain generic graphics and representations of the systems. As an example, Fig. 9 shows an output window constructed in the view part.

(3) Control: This part contains the actions that a user can perform on the simulation. A complete Graphical User Interface (GUI) can be constructed easily. A part from traditional actions like start, stop and reset; the control part allows to introduce interactivity. Fig. 7 and Fig. 8 show two windows containing different interactive which allow to tune and control the simulations elements and components.

This decomposition allows simplifying the application design and maintenance once it is ready. All three parts can be developed at high level without writing a single line of code ${ }^{1}$.

The IVL is composed by a set of windows where the user can parameterize and tune the system and the observe, analyze and compare the simulation results. The main window of the virtual lab is shown in Fig. 7, and it contains:

- Start, Stop and Reset buttons.

- Configuration menu, which allows to choose the configuration of the HEV (series, parallel or conventional) and the selected topology is also depicted.

- Speed profile, with a pointer to follow the current position in the profile.

- Dynamic bars which indicate the battery and ultracapacitor levels, and they corresponding values.

- Load selector.

Additional windows have been introduced in order to tune and analyze the energy management algorithms, as an example Fig. 8 has been designed in order to tune braking police. Once the desired configuration and the energy management has been selected the simulation can be initiated. The evolution of most relevant parameters can be observed in several graphical windows(Fig. 9). The applications allow to compare the results of different simulations obtained with different configurations or parameters, this can be used to analyze the effect of the different parameters or topologies.

\section{CONCLUSIONS}

This paper presents an IVL on an energy management systems, the Hybrid Electric Vehicle case. This IVL has been developed as a pedagogical tool to introduce engineering students in concepts and main ideas behind hybrid vehicles.

1 When needed JAVA code can be inserted in the system
First, the adopted model is described, which has been adopted as simple as possible in order to reduce the computational time. Then, the appearance and the interactive elements have been also presented. The presented laboratory contains interactive elements to provide the user different options: besides the HEV configuration, the power management policy can also be modified.

The use and evaluation of the virtual lab in courses of management of power systems will give a necessary feedback to improve the presented tool. Besides, future developments of the presented laboratory includes a pollution model to evaluate the effect on the emissions, and a power management tool with a more realistic behavior. The use of EJS with other kind of energy systems such as wind turbines, flywheel energy storage system, regenerative system for an urban train,... is also considered.

\section{ACKNOWLEDGEMENTS}

This work were partially supported by the Spanish government (DPI2007-62582), and the AGAUR (2007MQD00046) projects.

\section{REFERENCES}

A.F. Burke. Batteries and ultracapacitors for electric, hybrid, and fuel cell vehicles. Proceedings of the IEEE, 95(4):806-820, 2007.

C.C. Chan. State of the art of electric and hybrid vehicles. Proc. of the IEEE, 90(2):247-275, 2002.

S. Dormido, S. Dormido, R. Dormido, J. Sánchez, and N. Duro. The role of interactivity in control learning. International Journal of Engineering Education, 21(6): 1122-1133, 2005.

M. Ehsani, Y. Gao, S.E. Gay, and A. Emadi. Modern Electric, Hybrid Electric, and Fuel Cell Vehicles: Fundamentals, Theory, and Design. CRC, Boca Raton, FL, 2004.

M. Ehsani, Y. Gao, and J.M. Miller. Hybrid electric vehicles: Architecture and motor drives. Proc. of the IEEE, 95(4):719-728, 2007.

F. Esquembre. Creacion de simulaciones interactivas en Java. Pearson Educación - Prentice Hall, 2005. ISBN 8420540099.

Francisco Esquembre. Easy java simulations: a software tool to create scientific simulations in java. Computer Physics Communications, 156(2):199-204, January 2004.

J.M. Miller. Propulsion systems for hybrid vehicles. IEE, Power and Energy series, 2004.

J. Sánchez, S. Dormido, and F. Esquembre. The learning of control concepts using interactive tools. Computer Applications in Engineering Education, 13(1):84-98, 2004.

A. Sciarretta and L. Guzzella. Control of hybrid electric vehicles. IEEE Control Systems Magazine, 27(2):60-70, 2007.

John Shen, Abul Masrur, Vijay K Garg, and John Monroe. Automotive electric power and energy management - a system approach. BUSINESS BRIEFING : GLOBAL AUTOMOTIVE MANUFACTURING \& TECHNOLOGY, 2003. 